\title{
Longitudinal and Shear Wave Velocities Measurement in Compacted Bentonite for Water Content
}

\author{
Shun Kimura ${ }^{1,{ }^{*}}$, Kazumi Kitayama ${ }^{1}$, Hideharu Takahashi $^{1}$, Kazushi Kimoto $^{2}$, Katsuyuki Kawamura ${ }^{1}$, and \\ Hiroshige Kikura ${ }^{1}$ \\ ${ }^{1}$ Tokyo Institute of Technology, Tokyo, Japan \\ ${ }^{2}$ Okayama University, Okayama, Japan
}

\begin{abstract}
Bentonite is a good candidate of buffer material for disposal repository of high-level radioactive waste. Understanding groundwater behavior in bentonite buffer material is important in order to evaluate the bentonite buffer performance and guarantee long-term safety. Elastic constants of the bentonite buffer material are important parameters for the long-term safety. Water content in buffer material may have an influence on its elastic properties. For this reason, the monitoring system of the water saturation level in compacted bentonite is required. In this study, the ultrasonic velocity measurement method for evaluation of water content in compacted bentonite was proposed. At first, the effect of a degree of saturation in compacted bentonite on the longitudinal and shear wave velocities was investigated experimentally. In addition, the elastic property, bulk modulus, in unsaturated compacted bentonite were evaluated by ultrasonic velocities. As a result, it can be confirmed that ultrasonic velocities can evaluate a degree of saturation and bulk modulus of compacted bentonite.
\end{abstract}

\section{Introduction}

High-level radioactive waste (HLW) management is one of the important issues of nuclear power. HLW should be isolated from the human environment for more than 10,000 years because of its high radioactivity and long half-life. Disposal repository of HLW is designed to dispose of the waste in the deep underground with engineered and natural geological barriers as shown in Figure 1. The engineered barriers consist of the vitrified glass, overpack, and buffer material. Buffer material provides stable chemical and physical environment for inner engineered barriers. Bentonite, one of a clay mineral, is considered to be buffer material since it has low water permeability, low diffusion for nuclides, and good swelling property. Bentonite is planned to be compacted and installed in the disposal repository. Compacted bentonite buffer is unsaturated at the beginning of the waste management, and a degree of saturation will increase with time. Replenishment of water is called resaturation. Understanding the resaturation behavior inside compacted bentonite buffer is important because the water in bentonite has a tremendous effect on reactions between bentonite and radionuclides. The elastic properties of compacted bentonite have a dependency on a variation of water content. The elastic properties can be obtained by longitudinal and shear wave velocities. It means the variation of water content will be evaluated as a function of these wave velocities. Ultrasound is one of the elastic waves and is a useful technique for the laboratory experiments. Recently, ultrasonic velocity in compacted bentonite has been investigated for laboratory experiments [1], and seismic imaging technique for nonintrusive monitoring of the barrier in repositories [2] has been studied. For these reasons, in this study, the non-destructive ultrasonic measurement technique for monitoring of water saturation level in compacted bentonite was developed in our laboratory. Longitudinal and shear wave velocities measurement was conducted in order to investigate the relation between the velocities and water content in bentonite. In addition, bulk modulus, one of the elastic constant, was evaluated by the velocities.

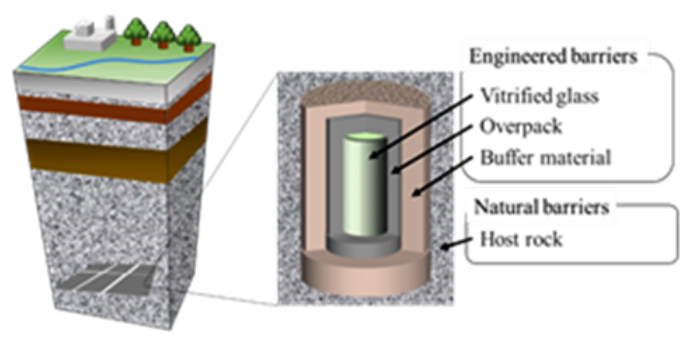

Fig.1. Schematic of disposal repository design of HLW.

\section{Experimental methodology}

\subsection{Sample preparation}

*Corresponding author: kimura@,us.nr.titech.ac.jp 
The sample material of pure smectic bentonite powder (Kunipia-F) was prepared for the compacted specimen. Kunipia-F is a purified bentonite and a content of a smectite, the main component of bentonite, is more than 99\%. Table 1 shows the chemical composition of Kunipia-F. The procedure of preparation was as follows. Bentonite was dried out for 24 hours at the temperature of $120^{\circ} \mathrm{C}$. Pure water was added to the dried bentonite to adjust water content. Water content $W$. is defined as

$$
W_{c}=\frac{M_{w}}{M_{s}}
$$

Where $M_{w}$ is the mass of water and $M_{s}$ is the mass of bentonite. The sample has four levels of water content: $\sim 20 \%, \sim 25 \%, \sim 30 \%$, and $\sim 35 \%$. Then, the bentonite powder is put into a cylindrical mold, and is compacted from both ends of the mold. Specimens have a diameter of $28 \mathrm{~mm}$ and a length of from $10 \mathrm{~mm}$ to $16 \mathrm{~mm}$. Dry density of specimens is between $1.00 \mathrm{~g} / \mathrm{cm}^{3}$ to 1.70 $\mathrm{g} / \mathrm{cm}^{3}$. In this study, the water content in compacted bentonite was evaluated by a degree of saturation. A degree of saturation can be calculated as follows:

$$
S_{r}=\frac{W_{c} \cdot \rho_{s}}{e \cdot \rho_{w}}
$$

Where $e, \varrho_{*}$, and $\varrho$ are a void ratio, density of water content and density of bentonite, respectively. Figure 2 shows the water content and the dry density of bentonite specimens. Red circles, green circles, blue circles and purple circles indicate four levels of bentonite, $\sim 20 \%$, $\sim 25 \%, \sim 30 \%$, and $\sim 35 \%$, respectively. The black line represents the fully saturated condition, a degree of saturation of $100 \%$.

Table 1. Chemical composition of Kunipia-F [3]

\begin{tabular}{|c|c|}
\hline $\mathrm{SiO} 2$ & 58.0 \\
\hline $\mathrm{TiO} 2$ & 0.2 \\
\hline $\mathrm{Al}_{2} \mathrm{O}_{3}$ & 21.9 \\
\hline $\mathrm{Fe} 2 \mathrm{O} 3$ & 1.9 \\
\hline $\mathrm{MnO}$ & 0.2 \\
\hline $\mathrm{MgO}$ & 3.4 \\
\hline $\mathrm{CaO}$ & 0.5 \\
\hline $\mathrm{Na} 2 \mathrm{O}$ & 3.0 \\
\hline $\mathrm{K} 2 \mathrm{O}$ & 0.1 \\
\hline $\mathrm{H} 2 \mathrm{O}$ & 10.8 \\
\hline
\end{tabular}

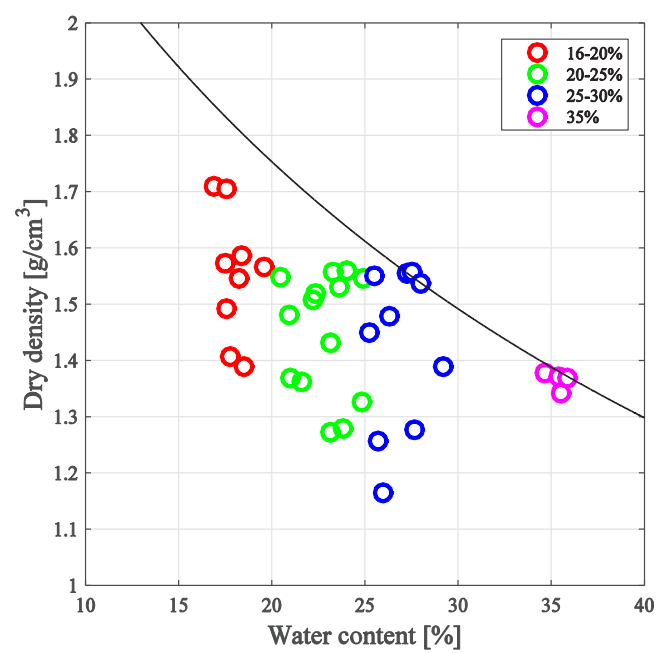

Fig.2. The water content and the dry density of bentonite specimens. Red circles, green circles, blue circles, and purple circles are water content of $\sim 20 \%, \sim 25 \%, \sim 30 \%$, and $\sim 35 \%$, respectively.

\subsection{Experimental setup}

Figure 3 shows the experimental apparatus for ultrasonic velocity measurement. The experimental apparatus consists of two ultrasonic transducers for longitudinal wave (B0.5C20N, Japan Probe Co., Ltd.), two ultrasonic transducers for shear wave $(1 \mathrm{Z} 10 \times 10 \mathrm{SN}$, Japan Probe Co., Ltd.), a pulser/receiver (JPR-10CN, Japan Probe Co., Ltd.), a computer, and an external amplifier (PR-60A5, Japan Probe Co., Ltd.). The transducer for longitudinal wave has an element with a diameter of $20 \mathrm{~mm}$. The center frequency of the transducer is $500 \mathrm{kHz}$. The transducer for shear wave has an element with a size of $10 \times 10 \mathrm{~mm}$. The transducer for shear wave has the center frequency of $1 \mathrm{MHz}$. Transducers were installed in opposite position at both ends of a specimen. The pulser/receiver was used for a transducer control by changing voltage and center frequency of the emitting wave. The frequency of the emitting wave was $500 \mathrm{kHz}$. Ultrasonic wave was emitted into the specimen from a surface and was received on an opposite surface.

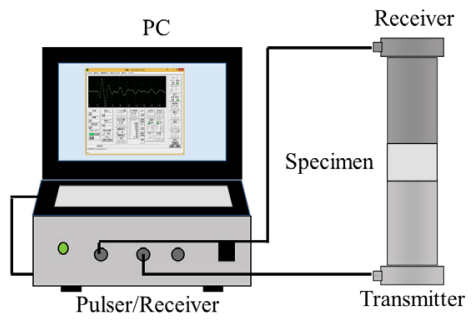

Fig.3. Schematic of the ultrasonic velocity measurement apparatus.

\subsection{Group velocity determination}

The basic theory of ultrasonic velocity measurement is based on the transit time from the emitting transducer 
to the receiving transducer. The velocity can be obtained by the propagation length and the transit time in Eq. (3).

$$
v=\frac{L}{\Delta t}
$$

Where $v$ is the ultrasonic velocity. $L$ is the propagation length of the ultrasonic wave between the emitting transducer and the receiving transducer. $\Delta t$ is the transit time.

The simplest method is the direct measurement of the first arrival of the received wave [4]. This method identifies the travel time from the time value of the first inflection of the receiving wave. Another typical method is a cross-correlation of emitting and receiving wave [4]. The peak at a time shift of a cross-correlation function indicates the transit time in this method. However, bentonite has a high attenuation of ultrasonic wave with frequency dependency. This attenuation causes the waveform distortions in the time domain. These time domain techniques are not applicable due to the attenuation of the ultrasonic wave in compacted bentonite. Therefore, frequency domain method based on Fourier transform was proposed. A signal in the time domain can be separated into Fourier amplitude and phase spectrum. The Fourier transform of a receiving signal is represented in Eq. (4).

$R(\omega)=\int_{-\infty}^{+\infty} r(t) e^{-i \omega t} d t=|R(\omega)| e^{-i \phi_{r}(\omega)}$

Where $\omega$ is angular frequency. $R(\omega)$ is Fourier transform of the receiving signal. $|R(\omega)|$ and $\varphi(\omega)$ are Fourier amplitude and the phase spectrum of the signal, respectively.

The attenuation in the time domain is caused by the attenuation of Fourier amplitude or the variation of the phase spectrum. Here, the variation of the phase spectrum in this measurement is not assumed. The differentiation of phase spectrum, the slope of phase spectrum, indicates the group delay. The group delay corresponds to the transit time of the signal. In order to obtain the transit time, the calibration of the initial phase using the signal of reference specimen is required. The polycarbonate specimen was used as the reference specimen. The transit time represents in Eq. (5).

$$
t_{g}(\omega)=\frac{d}{d \omega}\left(\phi_{r}-\phi_{p}\right)
$$

Where $\varphi_{s}(\omega)$ is the phase spectrum of polycarbonate. $t_{s}$ is the calibrated transit time. The calculated velocity in this method is called group velocity.

\subsection{Elastic properties}

Variations of water content may affect elastic properties of compacted bentonite. Elastic properties are characterized by elastic constants. Bulk modulus is one of elastic constants and defines resistance to compression. Therefore, bulk modulus means incompressibility of a material. Bulk modulus can be calculated from longitudinal wave velocity and shear wave velocity.

$$
K=\frac{\rho\left(3 V_{L}^{2}-4 V_{s}^{2}\right)}{3}
$$

Where $K$ is bulk modulus. $\varrho$ is a density of a material. $V_{L}$ and $V_{s}$ indicate longitudinal wave velocity and shear wave velocity, respectively. In this ultrasonic velocity measurement, the relation between a degree of saturation and bulk modulus was investigated.

\section{Results and discussions}

Figure 4 and 5 show examples of the receiving longitudinal wave, Fourier amplitude and phase spectrum of the reference specimen and compacted bentonite, respectively. The original signal was filtered by Hanning window in order to separate the first peak in Fig. 4 (a) and 5 (a). The black line indicates the original receiving wave and the red line indicates the filtered wave. Spike noises appear below $5 \mu$ s in receiving wave. These noises caused by emitting ultrasonic wave. The receiving wave cannot be distinguished in this region. Comparing Fourier amplitude in Fig. 4 (b) and Fig. 5 (b), it is can be found that the amplitude in compacted bentonite is attenuated strongly at a frequency of 300 $\mathrm{kHz}$ or more. Figure 6 shows an example of the calibrated transit time of longitudinal wave. The calibrated transit time can be calculated from the phase spectrum of reference specimen and compacted bentonite in Fig.4 (c) and Fig.5 (c). The calibrated transit time was obtained by averaging the group delay between $100 \mathrm{kHz}$ to $500 \mathrm{kHz}$.

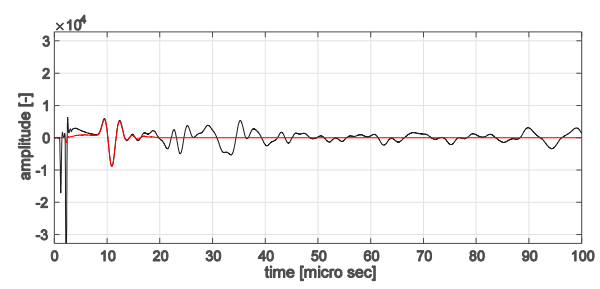

(a) Receiving wave

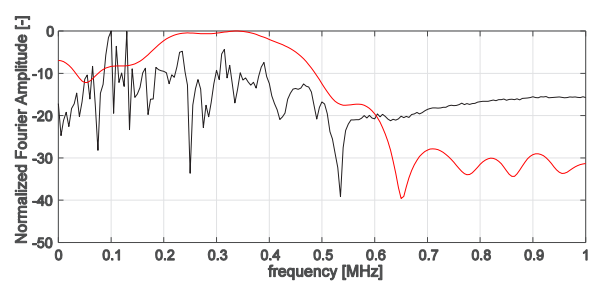

(b) Fourier amplitude.

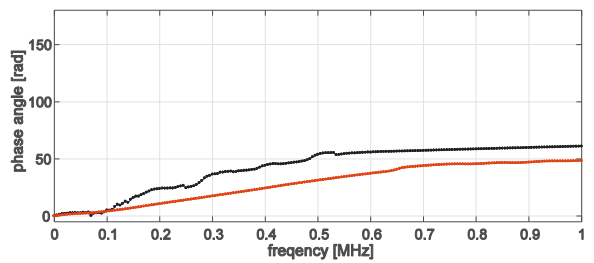

(c) Phase spectrum.

Fig.4. Receiving longitudinal wave and Fourier transform of the reference specimen. 


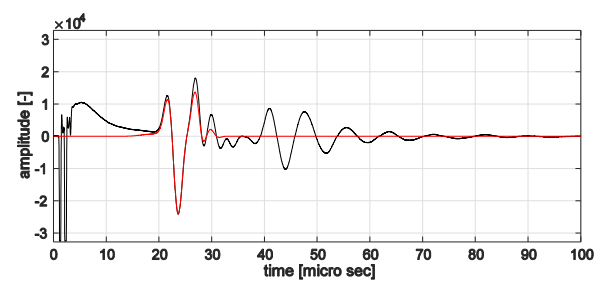

(a) Receiving wave.

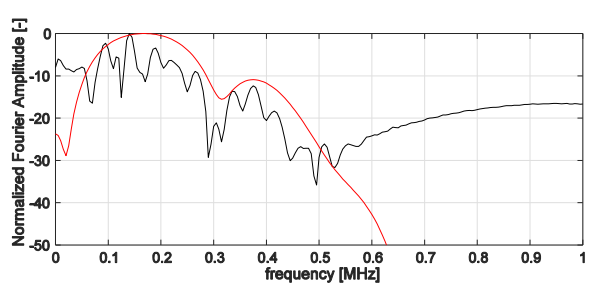

(b) Fourier amplitude.

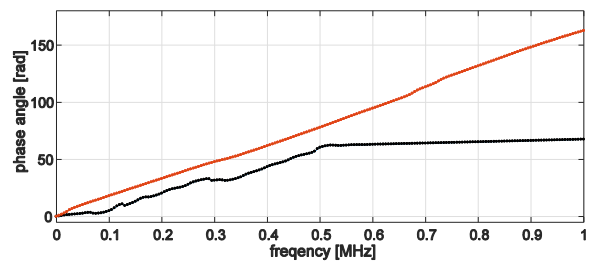

(c) Phase spectrum.

Fig.5. Receiving longitudinal wave and Fourier transform of compacted bentonite.

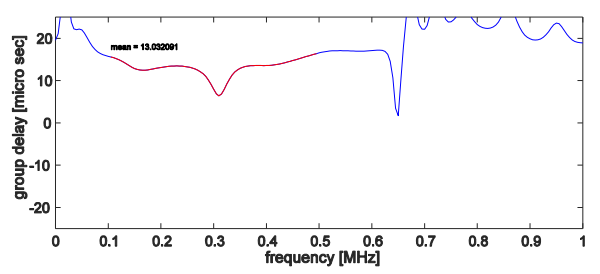

Fig.6. The calibrated transit time of the longitudinal wave.

Figure 7 shows the variation of longitudinal wave velocity with varying a degree of saturation. Red circles, green circles, blue circles, and purple circles are water content of $\sim 20 \%, \sim 25 \%, \sim 30 \%$, and $\sim 35 \%$, respectively in Fig.7. These colors in Fig.7 correspond to the colors in Fig.2. The longitudinal wave velocity increases with increasing the degree of saturation. It can be found that the velocity at a degree of saturation of $100 \%$ is very close to at the longitudinal wave velocity of $1,480 \mathrm{~m} / \mathrm{s}$ in water. Furthermore, the longitudinal wave velocity at low water content level is higher than high water content level in the same degree of saturation.

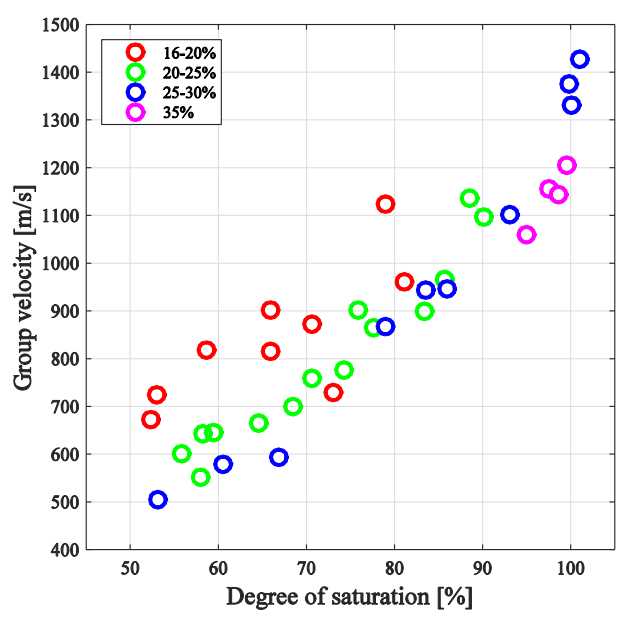

Fig.7. The variation of longitudinal wave velocity with varying a degree of saturation.

Figure 8 and 9 show examples of the receiving shear wave, Fourier amplitude and phase spectrum of the reference specimen and compacted bentonite, respectively. The black line represents the original receiving wave and the red line represents the filtered wave by Hanning window. Comparing with the shear wave of the reference and compacted bentonite, the waveform of compacted bentonite is distorted obviously. The distortion of the wave occurred due to the attenuation at a frequency around $500 \mathrm{kHz}$ as shown in Fig.9 (b). It can be confirmed that the identification of the first arrival of the distorted wave like in Fig.9 (a) is difficult. However, the phase spectrum of shear wave is approximately linear with frequency. Figure 10 shows an example of the calibrated transit time of shear wave. The calibrated transit time of the shear wave was obtained by averaging the group delay between $300 \mathrm{kHz}$ to $500 \mathrm{kHz}$.

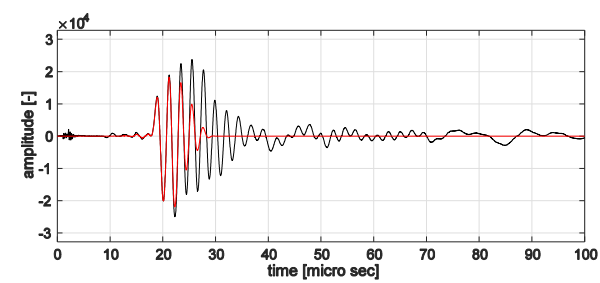

(a) Receiving wave.

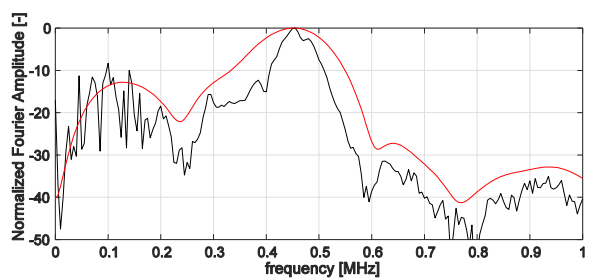

(b) Fourier amplitude. 


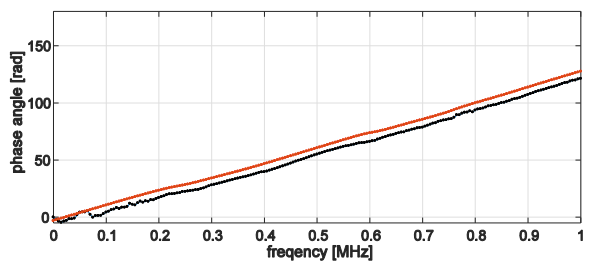

(c) Phase spectrum.

Fig.8. Receiving shear wave and Fourier transform of the reference specimen.

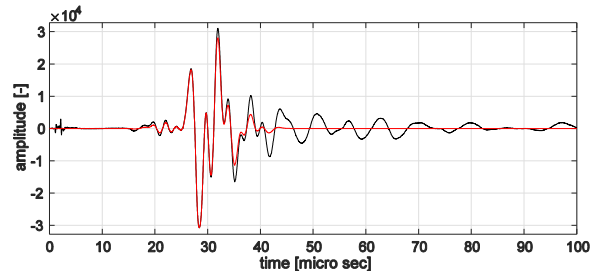

(a) Receiving wave.

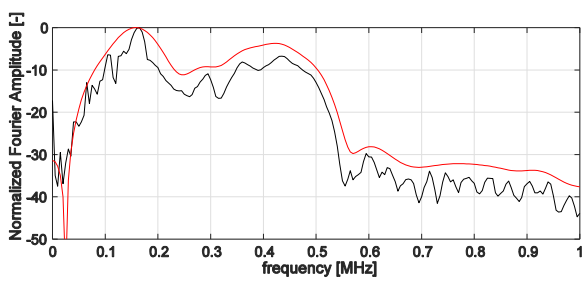

(b) Fourier amplitude.

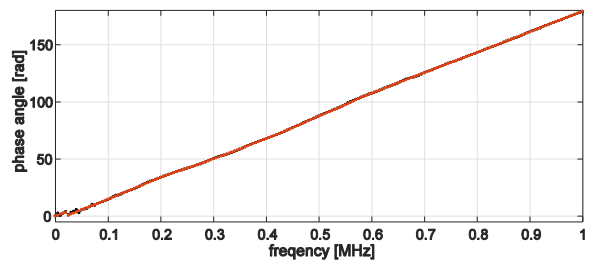

(c) Phase spectrum.

Fig.9. Receiving shear wave and Fourier transform of compacted bentonite.

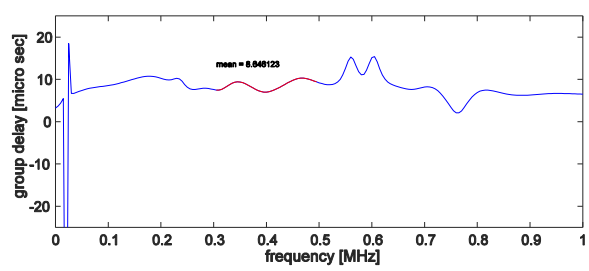

Fig.10. The calibrated transit time of the shear wave.

Figure 11 shows the variation of shear wave velocity with varying a degree of saturation. The shear wave velocity increases with increasing the degree of saturation. The velocity increases by $\sim 200 \mathrm{~m} / \mathrm{s}$ over $50 \%$ to $100 \%$ range. It can be seen that the water in compacted bentonite has a significant effect on longitudinal wave velocity than shear wave velocity.

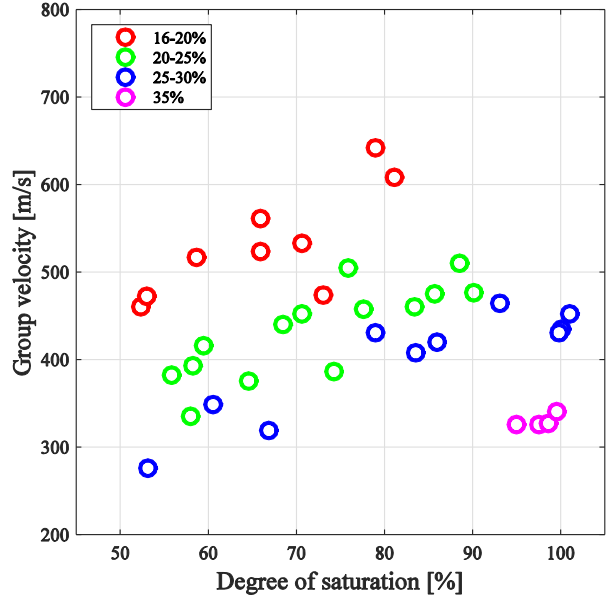

Fig.11. The variation of shear wave velocity with varying a degree of saturation.

The bulk modulus of compacted bentonite was calculated from measured longitudinal and shear wave velocities. Figure 12 shows the variation of the bulk modulus with varying a degree of saturation. The higher the degree of saturation increases the bulk modulus. In addition, the bulk modulus indicates almost the same value for all water content level in the same degree of saturation. Therefore, it is suggested that bulk modulus of compacted bentonite depends only on a degree of saturation. It means a degree of saturation in compacted bentonite can be evaluated by the bulk modulus.

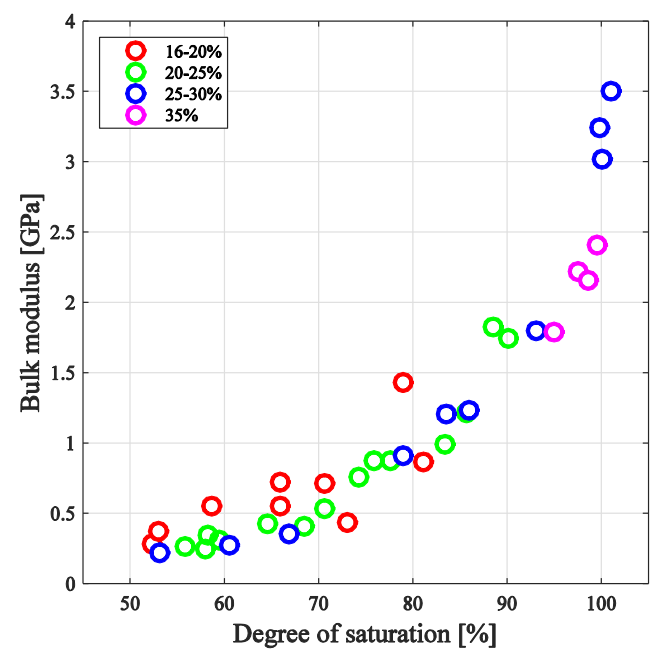

Fig.12. The variation of the bulk modulus with varying a degree of saturation.

\section{Conclusions}

The non-destructive ultrasonic measurement technique for monitoring of water saturation level in compacted bentonite was proposed. Longitudinal and shear wave velocities measurement for water content investigation was carried out. Frequency domain method 
was applied to the wave velocity calculation. The relation between longitudinal and shear wave velocities and a degree of saturation was investigated. Then, the bulk modulus of compacted bentonite was evaluated from measured velocities. Consequently, conclusions are as follows:

1. The ultrasonic wave attenuation in compacted bentonite was observed. In particularly, shear wave was distorted strongly. However, the frequency domain method for transit time determination can be applied in the high attenuation material like compacted bentonite. It was confirmed that this method can measure longitudinal and shear wave velocities in compacted bentonite.

2. Longitudinal and shear wave velocities increase with increasing a degree of saturation. The tremendous effect of water in compacted bentonite on longitudinal wave velocity was confirmed. The longitudinal velocity for fully saturated condition is very close to the velocity in water of $1,480 \mathrm{~m} / \mathrm{s}$.

3. The bulk modulus of compacted bentonite was evaluated from measured wave velocities. The higher the degree of saturation increases the bulk modulus. The bulk modulus of compacted bentonite depends only on a degree of saturation. It can be seen a degree of saturation in compacted bentonite can be evaluated by the bulk modulus.

\section{References}

1. N. Tisato, S. Marelli, J. Geophys. Res.: Solid Earth 118, pp. 3380-3393 (2013)

2. S. Marelli, E. Manukyan, E. Maurer, S.A. Greenhalgh, A.G Green, Geophysics, 75, Q21-Q34 (2010)

3. H. Sato, T. Ashida, Y. Kohashi, M. Yui, N. Sasaki, J. Nucl. Sci. Technol., 29, pp. 873-882 (1992).

4. A. Viana da Fonseca, C. Ferreira, M. Fahey, Geotech. Test. J., 32, pp. 91-107 (2009). 